\title{
Delays and diversity in the practice of local research ethics committees
}

\author{
Ala'Eldin H Ahmed and Karl G Nicholson Department of Microbiology and Immunology, School of \\ Medicine, Leicester University, Leicester.
}

\section{Abstract}

Objectives-To compare the practices of local research ethics committees and the time they take to obtain ethical approval for a multi-centre study.

Design- $A$ retrospective analysis of outcome of applications for a multi-centre study to local research ethics committees.

Setting-Thirty-six local research ethics committees covering 38 district health authorities in England. Main measures-Response of chairmen and women, the time required to obtain approval, and questions asked in application forms.

Results-We received replies from all 36 chairmen contacted: four (11\%) granted their approval, and 32 (89\%) required our proposal to be considered by their local research ethics committee. Three committees asked us to attend their meetings. The application was approved by all 36 local research ethics committees but the time to obtain ethical approval varied between six to 208 days. One third of the committees did not approve the project within three months, and three took longer than six months. There was considerable variation in the issues raised by local research ethics committees and none conformed exactly to the Royal College of Physicians' guidelines.

Conclusion-Obtaining ethical approval for a multicentre study is time-consuming. There is much diversity in the practice of local research ethics committees. Our data support the recommendation for a central or regional review body of multi-centre studies which will be acceptable to all local research ethics committees.

\section{Introduction}

Local research ethics committees (LRECs) in Britain developed following the Royal College of Physicians' (RCP) recommendations in 1967, which were subsequently disseminated by the Ministry of Health. ${ }^{12}$ Further guidance was provided by the RCP in 1973 and endorsed by the Department of Health in $1975 .{ }^{3}$ The RCP has updated its

\section{Key words}

Local research ethics committees; multi-centre study; ethical approval. guidance, ${ }^{4-6}$ and additional discussions and guidance on ethical issues relevant to medical research have been published in Britain. ${ }^{7-15}$ None the less surveys of LRECs suggest that the guidelines have had little impact on the composition and practice of the LRECs. ${ }^{16-19}$ Multi-centre studies have become increasingly more common, with a recent survey revealing that they represent $18 \%$ of the workload of LRECs. ${ }^{20}$

Because of the ever-increasing necessity for national and international multi-centre studies, it is essential that patients' interests are safeguarded and ethical approval obtained in the most efficient way. We report our experiences of obtaining ethical committee approval for a multi-centre Department of Health study which involved reviewing case-notes of deceased patients and controls.

\section{Method}

The Department of Public Health Medicine in Leicester initiated a retrospective review of general practitioners' (GP) notes of patients dying with influenza during the $1989 / 90$ epidemic to establish their vaccination status. Ethics committee approval was not considered necessary for this initial review and the results were subsequently published and formed the basis of a Department of Health multicentre, case-control study which started in December 1992. For the Department of Health case-control study we obtained ethical approval from the Leicestershire Committee on the Ethics of Clinical Research Investigation. The project involved identifying GP notes of patients whose deaths during the winter of $1989 / 90$ were certified as influenzal. Their notes and those of age- and sexmatched controls were to be examined to establish the influenza immunisation rates, with the goal of identifying whether vaccination protected against death from influenza. The project involved 40 district health authorities (DHAs) covering six health regions in England. The choice of the DHAs was dictated by whether the corresponding family health services authority (FHSA) had retained GP case notes of patients who died during the 1989/90 influenza epidemic. Two DHAs stated that ethical 
approval was not required for the proposed research. During the period December 1992 to July 1993 we sent a letter to the chairmen of 36 LRECs covering the remaining $38 \mathrm{DHAs}$, explaining the background leading to the proposed research, the aims and objectives of the project, and methods to be employed. A copy of the publication arising from the pilot study was included with the letter together with a copy of the questions to be addressed. We informed three chairmen that local ethical committee approval for this project was awaited. The remaining chairmen were contacted after approval in Leicester was obtained. We asked whether ethical approval was required in addition to that obtained in Leicester and if so, for a copy of the application form.

\section{Results}

\section{Response of the chairmen of LRECs}

A total of 36 chairmen of LRECs were contacted and replies were received from all 36 . Four $(11 \%)$ granted chairman's approval, but two of the four required completion of their LREC application form. One of the four required additional information, and chairman's approval was subsequently granted. The remaining 32 chairmen (89\%) required our proposal to be considered by their LREC, and 19 (59\%) wanted us to complete their local application forms, a copy of which was sent with the reply. (Altogether 21 committees wanted us to complete their local application forms). Of the 32 , three committees asked us to attend their meetings to discuss the proposal.

\section{Questions asked in LRECs' application forms}

Analysis of 20 of the 21 LREC application forms: (one application form was for questionnaire-based

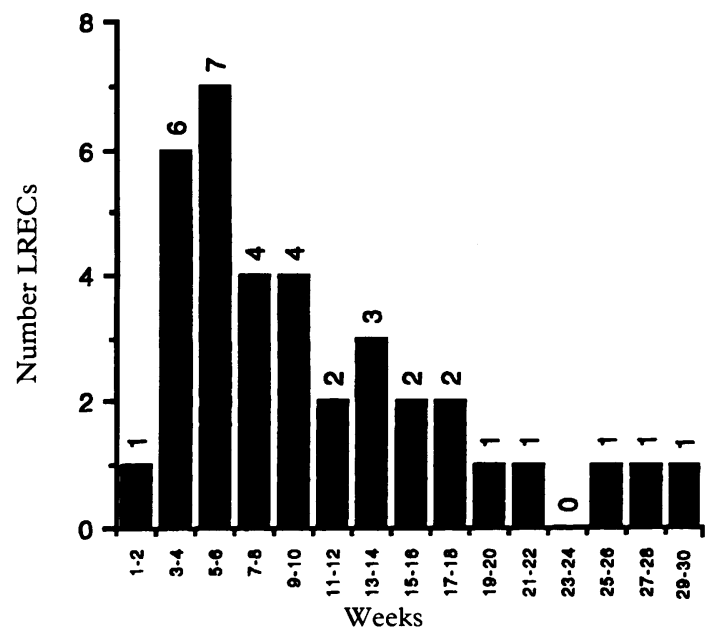

Figure. The interval between sending applications to 36 research ethics committees and being granted approval. research, and was not included in the analysis) revealed considerable variation in the number and type of questions asked. None conformed exactly to the suggested format for applications to research ethics committees as proposed by the $\mathrm{RCP}^{5}$ (see table). Only two (10\%) LRECs included questions relating to paediatric research, and only two $(10 \%)$ referred to specially vulnerable groups. Three committees $(15 \%)$ required a statement on the personal experience of the applicant in the field of investigation concerned.

\section{Time taken (days) to obtain ethical approval}

The interval between sending applications to 36 LRECs and receiving approval ranged from six to 208 days. The interval between sending the application and obtaining chairman's approval averaged 35 days (range six to 70 days) and for obtaining full committee approval averaged 77 days (range 18 to 208 days). The figure shows that one-third of the LRECs were unable to approve the project within three months, and three of the 36 LRECs took longer than six months.

Table Issues raised on ethical committee forms with reference to RCP guidelines

Issues

No of LRECs

State the title of the proposed project.

State the question to be answered and the value of answering it.

Give an outline of the proposed project including procedure, measurements, data analysis.

State the manner in which the subjects' consent will be obtained.

(percentage)

$20(100)$

20(100)

19(95)

Specify the type of subjects, how they will be recruited

State the potential hazards to subjects, and their estimated probability and the precautions to be taken to meet them.

State the procedures which may cause discomfort or distress.

If the project is designed to test a drug or appliance, state its exact regulatory status.

Attach any other relevant matter.

Is the study sponsored by an industrial company?

What arrangements, if any, for compensation in the event of injury to subjects have been made?

State any payments to subjects.

State the likely duration of the project and the premises in which it will be undertaken.

State any profit, personal or departmental, financial or otherwise, relating to the study.

State whether subjects' general practitioner is to be informed of recruitment of the subjects.

Specify whether subjects are in a dependent relationship with investigator.

State the personal experience of applicant in the field of investigation concerned.

Specify whether subjects are specially vulnerable, eg children, mentally handicapped.

Has the company provided a written statement that it accepts the current Guidelines of the association of the British Pharmaceutical Industry?

For paediatric projects: In what way can the proposed investigation be expected to benefit the individual patient, or a near relative.

If the investigation can not be expected to benefit the individual patient:
(a) are the risks minimal?
(b) Is parental or guardian agreement to be obtained?

(c) Is the child capable of giving assent? 


\section{Discussion}

The LRECs involved in this study may not have been a randomly selected sample, nevertheless they extend over a wide geographical area covering six health regions in England and hence reasonable conclusions can be drawn from this experience.

The response to our research proposal was diverse, ranging from no further ethical approval required, chairman's approval after completion of local research ethics committee application form, chairman's approval after provision of additional information, consideration of the submission by the full committee, consideration of a completed local research ethics committee form, and actual attendance at the ethics committee meeting. There is room for some diversity among ethics committees because of the subjective nature of ethical considerations and local needs. Admittedly in some of the areas in which we encountered different practices there are no clear guidelines. For example, there are no strict rules on the frequency of LREC meetings or whether they should be attended by the investigators. However, in areas where guidelines exist, our analysis highlighted a great degree of variation in the information asked of researchers. The RCP guidelines were issued in 1990 yet at the end of 1992 none of the 20 LRECs application forms for ethical approval that we completed conformed exactly to the guidelines. Failure to follow issued guidelines has been discussed before, ${ }^{17-19}$ and improving communication by adopting a registration system for LRECs and training potential committee members have been suggested as solutions ${ }^{19}$ but have yet to be implemented.

Obtaining ethical approval is time-consuming in the absence of a central review body for multi-centre studies. Evidently most LREC chairmen do not grant approval for studies approved elsewhere, and require a full submission to be made for local consideration. Overall one-third of the LRECs were unable to approve the project within three months, and three of the $36(8 \%)$ took longer than six months. As none of the committees rejected our submission, or required modification, delays in obtaining approval evidently relate to the frequency with which ethics committees meet, and also their workload. Moreover, the process of identifying and locating chairmen of ethics committees, and obtaining and completing application forms was also timeconsuming and therefore expensive. The average cost, including photocopying, postage, telephone calls, travel and time of research worker was estimated at approximately $£ 25.5$ per district, amounting to more than $£ 900$ for the study. Had the study required approval throughout England and Wales the cost of obtaining ethical approval is estimated to be in excess of $£ 5,000$.

A central system for ethical approval of multicentre studies has been suggested in the recent Department of Health guidelines. ${ }^{21}$ However, the Department of Health guidelines also recom- mended that the right of individual committees to call for review of a proposal should be retained. Other suggestions include a system based on establishing a cooperative database of all LRECs in England. Researchers seeking ethical approval for a multi-centre study would submit the protocol to any two LRECs in the collaborating group. ${ }^{22}$ The two LRECs involved would be informed of the other's involvement and would be able to discuss difficulties amongst themselves. Once approval has been granted the researchers can then circulate the approval letter with a copy of the protocol to the other collaborating LRECs. However, LRECs would have the power of veto. ${ }^{22}$

Historically LRECs developed according to their own views. In the early stages of their development no specific guidelines on practices and methods were given because it was thought that strict rules of conduct would not be adaptable to local needs. They have undoubtedly played a significant role in safeguarding patients' interests, dealing with a significant workload amounting on some occasions to reviewing 400 submissions a year. ${ }^{5}$ The two cardinal assets of LRECs are their independence and their invaluable local knowledge and because of the latter there are, arguably, advantages to having decisions made locally. Suggestions have, however, been made to try to preserve the independence of the LRECs on the one hand, and to avoid undue delay and unnecessary administrative work on the other. ${ }^{20}$ Consensus has yet to be reached. There is no evidence that repetitive review by many LRECs will provide greater protection for patients. ${ }^{23} 24$ Difficulties encountered as a result of repetitive review were recently highlighted in a report where five of six committees demanded changes to the proposal; none of them asked for the same changes. ${ }^{24}$

\section{Workable solution}

A system based on the adoption of a standard application form for researchers seeking approval for multi-centre studies, and the setting up a central or regional committee to give conditional approval constitutes a workable solution in the present system. The central or regional committee should send a copy of the conditionally approved protocol or a summary thereof, together with its letter of approval, to all the LRECs involved. By virtue of receiving a copy of the full protocol or summary, LRECs will have sufficient understanding of the scientific basis of the research project and its aims. Such a system would reduce bureaucracy by substantially reducing the amount of paperwork and expenses involved in making multiple submissions. Moreover, it would reduce the burden on LRECs, whose members are usually hard-working and unpaid. The role of such a committee should focus 
on the ethics of research, yet embrace scientific and statistical considerations, and its duties should be extended to include both clinical and epidemiological projects. We feel that the decisions of a central or regional committee should be binding on LRECs. However, their right to modify consent forms and patient information may be retained, since these are areas where their invaluable local knowledge is advantageous. It is important for such a committee to be independent and therefore its funding should be arranged centrally. Its membership should include lay people and senior and experienced people of distinction in their field who may be nominated by: the General Medical Council, DoH, RCP, Royal College of Nursing, Medical Research Council, The Association of Medical Research Charities, and The Association of the British Pharmaceutical Industry.

\section{Conclusion}

Our limited experience indicated that there is much diversity in the practice of LRECs. Our data clearly support the need for a central or regional review process for multi-centre studies, but it is essential that it addresses the perceived needs of all ethical committees in the United Kingdom and that the process is periodically audited.

Ala'Eldin $H$ Ahmed, MRCP, is Department of Health Clinical Research Fellow and Karl G Nicholson, MD, FRCP, FRCPath, is Senior Lecturer in Infectious Diseases, at the Department of Microbiology and Immunology, School of Medicine, Leicester University.

\section{References}

1 Royal College of Physicians. Supervision of the ethics of clinical research investigations in institutions. London: HMSO, 1967 (updated 1973).

2 Ministry of Health. Supervision of the ethics of clinical research. London: HMSO, 1968: $\mathrm{HM}(68) 33$.

3 Department of Health and Social Security. Supervision of ethics of clinical research investigations and fetal research. London: HMSO, 1975: HSC (IS) 153.

4 Royal College of Physicians. Guidelines on the practice of ethics committees in medical research. London: RCP, 1984.

5 Royal College of Physicians. Guidelines on the practice of ethics committees in medical research involving human subjects. London: RCP, 1990.

6 Royal College of Physicians. Research on healthy volunteers. Fournal of the Royal College of Physicians of London 1986; 20: 243-7.

7 Diamond AL, Laurence DR. Compensation and drug trials. British Medical fournal 1983; 287: 675-7.

8 Joint committee of the Association of the British Pharmaceutical Industry, British Medical Association, Committee on Safety of Medicines, and Royal College of General Practitioners. Guidelines on postmarking surveillance. British Medical fournal 1988; 296: 399-400.

9 Working Party on Ethics of Research in Children. Guidelines to aid ethical committees considering research involving children. British Medical fournal 1980; 280: 229-31.

10 Medical Research Council. Responsibility in the use of personal medical information for research. London: MRC, 1985.

11 British Medical Association, Royal College of General Practitioners, and Association of the British Pharmaceutical Industry. Code of practice for the clinical assessment of licensed medicinal products in general practice. British Medical fournal 1983; 286: 1295-7.

12 Anonymous. Local ethical committees [editorial]. British Medical fournal 1981; 282: 1010.

13 Marshall T, Moodie P. Scrutinising research ethics committees. British Medical fournal 1988; 297: 753.

14 Department of Health and Social Security. Administration of radioactive substances to persons. London: HMSO, 1984. HN(84)5.

15 Harding NGL, ed. Data protection in medicine. Bristol: NHS Training Authority, 1986.

16 Neuberger J. Ethics and health care: the role of research? ethics committees in the United Kingdom. London: King's fund institute, 1992.

17 Nicholson R, ed. Medical research with children: ethics law and practice. Oxford: Oxford University Press, 1986.

18 Thompson IE, French K, Melia K, Boyd KM, Templeton AA, Potter B. Research ethics committees in Scotland. British Medical fournal 1981; 282: 718-20.

19 Gilbert C, Fulford KWM, Parker C. Diversity in the practice of district ethics committees. British Medical fournal 1989; 299: 1437-9.

20 Moran J. Local research ethics committees, report of the second national conference. Fournal of the Royal College of Physicians of London 1992; 26(4): 423-31.

21 Department of Health. Local research ethics committees. London: DoH, 1991.

22 Horwitz R. Ethical review of multi-centre research. Bulletin of Medical Ethics 1994; Oct: 13-5.

23 Berry TJ, Ades TE, Peckham CS. Too many ethical committees. British Medical fournal 1990; 301: 1274.

24 Hotopf M, Wessely S, Noah N. Are ethical committees reliable? fournal of the Royal Society of Medicine 1995; 88: $31-3$. 